POS PROCEEDINGS

\title{
KKLT and the Swampland Conjectures
}

\section{Ralph Blumenhagen*}

Max-Planck-Institut für Physik, Föhringer Ring 6, 80805 München, Germany

E-mail: blumenha@mpp.mpg.de

\section{Max Brinkmann}

Max-Planck-Institut für Physik, Föhringer Ring 6, 80805 München, Germany

E-mail: mbrinkm@mpp.mpg • de

\section{Daniel Kläwer}

PRISMA Cluster of Excellence and Mainz Institute for Theoretical Physics, Johannes

Gutenberg-Universität, 55099 Mainz, Germany

E-mail: klaewer@uni-mainz.de

\section{Andriana Makridou}

Max-Planck-Institut für Physik, Föhringer Ring 6, 80805 München, Germany

E-mail: amakrido@mpp.mpg.de

\section{Lorenz Schlechter}

Max-Planck-Institut für Physik, Föhringer Ring 6, 80805 München, Germany

E-mail: lschlech@mpp.mpg.de

Recently, various swampland conjectures have been proposed that every UV complete effective field theory should satisfy. In particular, these deal with the properties of AdS and dS solutions arising in theories of quantum gravity. Mainly summarizing the results of our two previous articles $[1,2]$, we confront the string theory based KKLT scenario with these conjectures and argue that if quantum vacua as in the KKLT construction indeed exist, some of the conjectures receive some log-corrections. Furthermore we point out some new aspects not contained in the aforementioned two papers.

Corfu Summer Institute 2019 "School and Workshops on Elementary Particle Physics and Gravity" (CORFU2019)

31 August - 25 September 2019

Corfu, Greece

\footnotetext{
*Speaker.
} 


\section{Introduction}

During the last years, the idea of a string or quantum gravity swampland [3,4] was further developed into various directions and in the moment can be considered the hot topic in the field of string phenomenology/cosmology. As opposed to the string theory landscape, the swampland contains those low-energy effective theories that cannot be UV completed to a consistent theory of quantum gravity. The aim of the swampland program is to extract a set of relatively simple features that low-energy effective field theories should satisfy in order to admit such an embedding into a theory of quantum gravity (see $[5,6]$ for recent reviews). This makes string theory much more predictive than previously expected from the picture of a vast string landscape.

Several such swampland conjectures have been proposed. In this article we focus on a number of them that mainly deal with the properties of anti-de Sitter (AdS) and de Sitter solutions in string theory ${ }^{1}$. First, these are the AdS/moduli scale separation conjecture (AM-SSC) [8] and the AdS distance conjecture (ADC) [9] (see also [10,11]). These conjectures say that the mass of certain (towers of) modes cannot be parametrically separated from the AdS radius. The strong version of the ADC is reminiscent of observations made earlier in [12]. Second there are conjectures that are related to dS solutions, namely the $\mathrm{dS}$ swampland conjecture [13-16] and its quantum version called the trans-Planckian censorship conjecture (TCC) [17]. This quantum generalization admits meta-stable dS extrema as long as their life-time is sufficiently small. In addition we also discuss the emergence proposal [18-20] of infinite distances in field space that is closely related to the swampland distance conjecture $[4,21]$.

These conjectures have been motivated and tested in the framework of tree-level constructions of AdS and dS vacua. However, it is well known that there exist also constructions of such vacua that utilize not only tree-level ingredients, but also quantum, in particular non-perturbative effects. The most famous examples are the KKLT [22] and the large volume scenario (LVS) [23]. In both cases, AdS minima are found in the effective 4D potential and subsequently uplifted to dS. This article is essentially a brief review of recent work on relating the KKLT construction to the aforementioned swampland conjectures.

In section 2 we briefly review the relevant AdS and dS swampland conjectures, followed in section 3 by the presentation of two prototypical AdS flux vacua arising in the class of type IIA orientifolds. Section 4 provides a description of the KKLT construction in the framework of an effective theory which takes into account that for a successful uplift, a strongly warped throat geometry is required. In fact, starting with [24] there were a couple of recent papers that deal with such a warped KKLT model both from a stringy $10 \mathrm{D}$ perspective $[1,25,26]$ and from an effective 5D point of view [27]. In this proceedings article we will mostly focus on our own work [1], where in particular the existence of an exponentially light tower of KK modes was established. This also fits nicely into the scheme of the emergence proposal. In section 5 we discuss the relation of the KKLT construction to the AdS and dS swampland conjectures. Taken at face value, the KKLT model does not satisfy the two AdS conjectures but would do so once one introduces quantum logcorrections to the swampland conjectures [2]. The quantum contributions found are reminiscent of a similar correction in the TCC. We also include some new aspects into this review article such as

\footnotetext{
${ }^{1}$ Recently, there was also a conjecture concerning Minkowski minima in string theory [7]. This says that in 4D theories with genuine $\mathscr{N}=1$ supersymmetry there are no exact Minkowski minima.
} 
a closer investigation of the dS saddle point that the KKLT model develops once one includes $\alpha^{\prime}$ corrections to the Kähler potential.

\section{Swampland conjectures}

In this section we briefly review some of the swampland conjectures that have been proposed. First, we consider swampland conjectures related to AdS and dS solutions. Second, we review the emergence proposal for (infinite) distances in field space.

\subsection{AdS and dS swampland conjectures}

While dS vacua are notoriously difficult to get in concrete string theory constructions, AdS solutions already exist at the classical level. The most famous example is certainly the $\operatorname{AdS}_{5} \times S^{5}$ solution of the type IIB superstring. We distinguish two conjectures that deal with the property of these AdS spaces. The first one gives a relation between the lightest (non-zero) moduli mass and the scale of the AdS space [8].

AdS/moduli scale separation conjecture (AM-SSC):

In an AdS minimum one cannot separate the size of the AdS space and the mass of its lightest mode. Quantitatively, they satisfy the relation

$$
m_{\text {mod }} R_{\text {AdS }} \leq c
$$

where $R_{\mathrm{AdS}}^{2} \sim|\Lambda|^{-1}$ and c denotes an order one parameter.

To our knowledge, this conjecture is satisfied in all known tree-level AdS flux vacua. We will see later that for the KKLT AdS minimum, relation (2.1) receives log-corrections. The second swampland conjecture is reminiscent of the swampland distance conjecture and relates the AdS scale to the mass scale of a tower of light states [9].

AdS distance conjecture (ADC):

For an AdS vacuum the limit $\Lambda \rightarrow 0$ is at infinite distance in field space and there is a tower of light states with

$$
m_{\text {tower }}=c_{\text {AdS }}|\Lambda|^{\alpha}
$$

for $\alpha>0$.

In the original paper [9], a stronger version was proposed saying that for supersymmetric AdS minima one always finds $\alpha=1 / 2$, effectively forbidding any scale separation of internal and AdS space. However, subsequent work [28-31] supports the original claim of DGKT [32] that supersymmetric type IIA flux compactifications provide a class of scale separated AdS vacua. As will be recalled in section 3, these models feature a four-form flux that is unbounded by any tadpole cancellation condition and leads to a KK scale $m_{\mathrm{KK}} \sim|\Lambda|^{7 / 18}$, hence violating the strong AdS distance conjecture. It was suggested in [2] that irrespective of supersymmetry the strong ADC holds for models where curvature terms are relevant for moduli stabilization. In the framework of flux 
compactifications this is described by turning on so-called geometric fluxes. The $A d S_{5} \times S^{5}$ background is of this type when described by an effective 5D theory. Another example will be presented in section 3.

The conjecture with probably the boldest cosmological implications, though stated as a general bound on the scalar potential in a theory of quantum gravity $[13,14]$, deals with the existence of dS vacua $^{2}$.

Refined dS swampland conjecture:

The scalar potential satisfies either of the two properties

$$
|\nabla V| \geq \frac{c}{M_{\mathrm{pl}}} \cdot V \quad \text { or } \quad \min \left(\nabla_{i} \nabla_{j} V\right) \leq-\frac{c^{\prime}}{M_{\mathrm{pl}}^{2}} \cdot V
$$

forbidding de Sitter minima.

Note that for AdS vacua the first condition is always satisfied. The second condition means that dS extrema are always saddle points that should decay fast enough. Indeed, the life-time of such a dS saddle point has been estimated in [17] as

$$
T \sim-\frac{3 H}{m_{\text {tach }}^{2}}
$$

where $m_{\text {tach }}^{2}$ is defined as the smallest negative eigenvalue of the mass ${ }^{2}$ operator, so we get the bound $T \leq\left(c^{\prime} H\right)^{-1}$. All stringy dS saddle points that we are aware of do indeed satisfy this conjecture. Note that for our universe this would mean that the life-time is shorter than its present life-time. Therefore, if this conjecture is correct, it seems that quintessence ${ }^{3}$ would remain the only natural candidate to explain the cosmological $\Lambda \mathrm{CDM}$ model $[35,36]$.

In looking for a conceptual explanation of the dS swampland conjecture, a slightly weaker form was proposed [17]. This is the trans-Planckian censorship conjecture (TCC), which states that sub-Planckian fluctuations should not become classical (in an expanding universe). Quantitatively it says

$$
\int_{t_{i}}^{t_{f}} d t H<\log \left(\frac{M_{\mathrm{pl}}}{H_{f}}\right)
$$

which implies a weaker bound on the life-time of a dS phase

$$
T \leq \frac{1}{H} \log \left(\frac{M_{\mathrm{pl}}}{H}\right)
$$

We think it would be interesting to identify stringy dS extrema that saturate this bound, i.e. including the log-factor. In this case, the present expansion of our universe could also be a consequence of a hill-top phase of rolling.

\footnotetext{
${ }^{2}$ Motivated by [33], initially in [13] only the first relation in (2.3) was proposed. This would exclude also dS saddle points that are believed to exist in the string theory landscape.

${ }^{3}$ See [34] for a compilation of the harsh observational bounds on stringy models of quintessence.
} 
The TCC implies a weaker form also for the first condition in the dS swampland conjecture (2.3). Namely, for a monotonically decreasing positive potential, it yields a global bound

$$
\left.M_{\mathrm{pl}}\left\langle\frac{-V^{\prime}}{V}\right\rangle\right|_{\phi_{i}} ^{\phi_{f}}>\frac{1}{\Delta \phi} \log \left(\frac{V_{i}}{A}\right)+\frac{2}{\sqrt{(d-1)(d-2)}}
$$

for the average of $-V^{\prime} / V$ in the interval $\left[\phi_{i}, \phi_{f}\right]$. Here $A=M_{\mathrm{pl}}^{4}(d-1)(d-2) / 2$ is a constant. In its asymptotic limit it provides a prediction of the parameter $c=2 / \sqrt{(d-1)(d-2)}$ in (2.3). Recently, the TCC was also related to the swampland distance conjecture $[37,38]$.

\subsection{Emergence proposal}

The emergence proposal [18-20] can be considered as an extension of the swampland distance conjecture ${ }^{4}$ [4]. The latter says that approaching points at infinite distance $\phi \rightarrow \infty$ in the moduli space of an effective field theory, a tower of states becomes exponentially light

$$
m_{n} \sim f(n) e^{-\lambda \phi}
$$

with $f(n)=n$ for KK towers, or $f(n)=\sqrt{n}$ for the denser weakly coupled string spectrum [40-43]. This leads to a breakdown of the effective action for $\phi>\lambda^{-1}$. Moreover, this critical scale is of the order of the Planck scale.

Emergence proposal:

The infinite distance in the IR appears from integrating out the tower of light states in the UV.

To make this more concrete, consider a light field $\phi$ and a tower of massive states $h_{n}$ with mass $m_{n}=n \Delta m(\phi)$ and degeneracy $\operatorname{deg}(n)=n^{K}$ governed by an effective action

$$
S=M_{\mathrm{pl}}^{D-2} \int d^{D} x\left(\frac{1}{2} G_{\phi \phi} \partial_{\mu} \phi \partial^{\mu} \phi+\sum_{n} \frac{1}{2} \partial_{\mu} h_{n} \partial^{\mu} h_{n}+\frac{1}{2} m_{n}^{2}(\phi) h_{n}^{2}\right)
$$

where $G_{\phi \phi}$ denotes the metric on field space. Integrating out the modes that are lighter than the species scale

$$
\Lambda_{\mathrm{sp}}=\frac{\Lambda_{\mathrm{UV}}}{N_{\mathrm{sp}} \frac{1}{D-2}}
$$

leads to a one-loop correction to the metric $G_{\phi \phi}$ as

$$
G_{\phi \phi}^{1-\text { loop }} \sim \frac{1}{M_{\mathrm{pl}}^{D-2}} \sum_{n=1}^{N_{\mathrm{sp}}}\left(\partial_{\phi} m_{n}(\phi)\right)^{2} \sim \frac{\Lambda_{\mathrm{sp}}^{D+K-1}}{M_{\mathrm{pl}}^{D-2}} \frac{\left(\partial_{\phi} \Delta m(\phi)\right)^{2}}{(\Delta m(\phi))^{K+3}} .
$$

The emergence proposal then calls for $G_{\phi \phi}^{1-\text { loop }} \sim G_{\phi \phi}^{\text {tree }}$. In its stronger form it even claims that the initial tree-level metric is vanishing in the $\mathrm{UV}$, and that it is entirely emergent from integrating out massive modes.

\footnotetext{
${ }^{4} \mathrm{~A}$ review talk on the relation of large field inflation in string theory and the swampland distance conjecture was given at the Corfu Summer School 2017 [39].
} 


\section{Tree-level flux vacua}

In this section we remind the reader of two classes of AdS minima that occur in tree-level flux compactifications of type IIA orientifolds. The first class are the so-called DGKT models [32,44] (see also [45] for more recent studies) for which solely various NS-NS and R-R $p$-form fluxes are turned on. In the second class one also allows geometric fluxes which can be considered as generalizations of the $A d S_{5} \times S^{5}$ Freud-Rubin type vacua.

As we will see, for properly deciding whether these well-known vacua abide by the conjectures, we need to identify the relevant scales. Concerning the subcase of the ADC for which the relevant tower of light states is the Kaluza-Klein one, we critically address the usual estimate for the KK scale $m_{\mathrm{KK}} \sim 1 / R$, where $R$ is the relevant length scale for the compactification manifold.

\subsection{Example I: Type IIA flux compactifications}

The special feature of this class of flux compactifications is that all closed string moduli can be stabilized at tree-level via R-R and $H_{3}$ form fluxes. In [9], it was noted that the DGKT models satisfy the ADC, but not in its strong version. Let us understand this claim through a particularly simple yet enlightening example. The compactification manifold we choose is the isotropic sixtorus $T^{6}$, which means that the moduli superfields will be identified as $T_{1}=T_{2}=T_{3} \equiv T$ and $U_{1}=U_{2}=U_{3} \equiv U$. So we have only three remaining superfields $S, T, U$. The real parts of the superfields are defined as

$$
\tau=r_{x} r_{y}, \quad s=e^{-\phi} r_{x}^{3}, \quad u=e^{-\phi} r_{x} r_{y}^{2} .
$$

The Kähler potential is as usual given by

$$
K=-3 \log (T+\bar{T})-3 \log (U+\bar{U})-\log (S+\bar{S}),
$$

while for the superpotential we make the restricted choice

$$
W=i f_{0} T^{3}-3 i f_{4} T+i h_{0} S+3 i h_{1} U,
$$

stabilizing all axions at vanishing value. The only non-vanishing contributions to the $C_{7}$-form tadpole come from the combinations $f_{0} h_{i}$ that in a full model need to be cancelled by the $O 6$-planes and $D 6$-branes. Thus, the four-form flux $f_{4}$ is not bounded by any topological condition.

Computing the scalar potential, one realizes that there exist both supersymmetric and nonsupersymmetric AdS minima. For instance in the supersymmetric vacuum, the moduli are stabilized at

$$
\tau=\kappa \frac{f_{4}^{\frac{1}{2}}}{f_{0}^{\frac{1}{2}}}, \quad s=\frac{2 \kappa}{3} \frac{f_{4}^{\frac{3}{2}}}{f_{0}^{\frac{1}{2}} h_{0}}, \quad u=2 \kappa \frac{f_{4}^{\frac{3}{2}}}{f_{0}^{\frac{1}{2}} h_{1}}
$$

with $\kappa=\sqrt{5 / 3}$. For the non-supersymmetric minima only the numerical prefactors change. The effective masses of the moduli all scale in same way as

$$
m_{\text {mod }}^{2} \sim-\Lambda \sim \frac{f_{0}^{\frac{5}{2}} h_{0} h_{1}^{3}}{f_{4}^{\frac{9}{2}}} M_{\mathrm{pl}}^{2} .
$$


Therefore the AM-SSC is satisfied, also in its strong form. Since we have two geometric radii, there will also exist two KK scales. Those are

$$
m_{\mathrm{KK}, 1}^{2}=\frac{M_{s}^{2}}{r_{x}^{2}}=\frac{M_{\mathrm{pl}}^{2}}{s \tau u}=\frac{f_{0}^{\frac{3}{2}} h_{0} h_{1}}{f_{4}^{\frac{7}{2}}} M_{\mathrm{pl}}^{2}, \quad m_{\mathrm{KK}, 2}^{2}=\frac{M_{s}^{2}}{r_{y}^{2}}=\frac{M_{\mathrm{pl}}^{2}}{\tau u^{2}}=\frac{f_{0}^{\frac{3}{2}} h_{1}^{2}}{f_{4}^{\frac{7}{2}}} M_{\mathrm{pl}}^{2} .
$$

Choosing the fluxes as $f_{0}, h_{0}, h_{1}=O(1), f_{4} \gg 1$, the values of the moduli are in the perturbative regime where the string coupling is weak $\left(g_{s} \ll 1\right)$ and the radii large. One notices that in this regime the KK scales are parametrically larger than the moduli masses and one has

$$
m_{\mathrm{KK}, i}^{2} \sim|\Lambda|^{\frac{7}{9}},
$$

thus satisfying the ADC with $\alpha=7 / 18$, for both the SUSY and non-SUSY vacua. Since $\alpha \neq \frac{1}{2}$, as noted in [9] the strong ADC is indeed violated. This mass scaling above reflects the original claim [32] that type IIA flux compactifications admit a dilute flux limit (where $f_{4} \rightarrow \infty$ ).

An important question is whether this effective $4 \mathrm{D}$ analysis can really be uplifted to a full solution of the 10D string equations of motion. The difficulty lies in the proper description of the localized O6-plane/D6-brane sources. A couple of important steps in this direction have been performed recently. In [29], the backreaction was taken into account in a perturbation around the known smeared solution. Another interesting idea was put forward in [30], where a refinement of the ADC was proposed that includes a discrete $\mathbb{Z}_{k}$ symmetry. Finally, in [31] approximate solutions to the 10D supersymmetry equations with similar features to the 4D DGKT vacua were found, satisfying a non-trivial test for the 4D vacua to admit a 10D uplift. All these approaches do not hit any obstacle for the scale separation of DGKT vacua to hold.

\subsection{Example II: Type IIA with geometric flux}

Let us now consider an example which is more similar to the Freund-Rubin case in the sense that there is also a geometric (curvature) contribution necessary for the stabilization of the moduli. Describing the $A d S_{5} \times S^{5}$ case from an effective 5D perspective ${ }^{5}$, a positive 5-form flux contribution is balanced against a negative curvature contribution. Such backgrounds can be described by turning on geometric flux $\omega$ in the effective theory. While many models of this type have been considered in [44], here we just focus on an illustrative example.

Like in the previous example, consider now a toroidal type IIA orientifold with the superpotential

$$
W=f_{6}+3 f_{2} T^{2}-\omega_{0} S T-3 \omega_{1} U T
$$

where the last two contributions arise from geometric fluxes. The scalar potential is found to have a supersymmetric AdS minimum at

$$
\tau=\frac{1}{3} \frac{f_{6}^{\frac{1}{2}}}{f_{2}^{\frac{1}{2}}}, \quad s=2 \frac{f_{2}^{\frac{1}{2}} f_{6}^{\frac{1}{2}}}{\omega_{0}}, \quad u=2 \frac{f_{2}^{\frac{1}{2}} f_{6}^{\frac{1}{2}}}{\omega_{1}},
$$

\footnotetext{
${ }^{5}$ Similar backgrounds of the form $A d S_{2} \times M^{(1)} \times \ldots \times M^{(n)}$, with $M^{(i)}$ Riemannian spaces, have been examined recently in [46].
} 
in which the moduli masses scale as

$$
m_{\mathrm{mod}}^{2} \sim-\Lambda \sim \frac{\omega_{0} \omega_{1}^{3}}{f_{2}^{\frac{1}{2}} f_{6}^{\frac{3}{2}}} M_{\mathrm{pl}}^{2} .
$$

In this case the two KK scales are

$$
m_{\mathrm{KK}, 1}^{2}=\frac{\omega_{0} \omega_{1}}{f_{2}^{\frac{1}{2}} f_{6}^{\frac{3}{2}}} M_{\mathrm{pl}}^{2}, \quad m_{\mathrm{KK}, 2}^{2}=\frac{\omega_{1}^{2}}{f_{2}^{\frac{1}{2}} f_{6}^{\frac{3}{2}}} M_{\mathrm{pl}}^{2}
$$

which satisfy $m_{\mathrm{KK}, 1}^{2} \sim \frac{m_{\mathrm{mod}}^{2}}{\omega_{1}^{2}}$ and $m_{\mathrm{KK}, 2}^{2} \sim \frac{m_{\mathrm{mod}}^{2}}{\left(\omega_{1} \omega_{2}\right)}$. Naively looking at this result, one would conclude that a suitable selection of fluxes allows for a parametric scale separation, although in the wrong direction $m_{\mathrm{KK}}<m_{\text {mod }}$.

However, as anticipated in [2] and concretely shown in [47], to draw such a conclusion it is important to take the backreaction of the fluxes onto the metric into account. Looking more carefully into the situation, one realizes that the actual eigenvalue problem one has to solve is

$$
\Delta_{\bar{G}} \chi(y)+\Phi(\bar{G}, \bar{F}, \ldots) \chi(y)=-m_{\mathrm{KK}}^{2} \chi(y)
$$

where $\bar{G}, \bar{F}$ denote the fully backreacted background metric and flux. When doing so, the geometric fluxes in the denominator also cancel and parametrically one indeed finds $m_{\mathrm{KK}}^{2} \sim m_{\mathrm{mod}}^{2} \sim|\Lambda|$. Therefore, in such models with geometric flux there is no parametric separation of the KK scale and the moduli masses. Note that this means that both in the supersymmetric and non-supersymmetric case the relation from the strong ADS conjecture holds.

Thus, a well motivated guess is that the backreaction is of paramount importance whenever geometric effects, such as geometric fluxes, contribute to the moduli stabilization. Moreover, we would like to suggest that the relevant criterion for the strong version of the ADS conjecture $(\alpha=$ $1 / 2$ ) to hold is not supersymmetry but whether geometric fluxes are involved when describing the model from the 4D effective field theory perspective.

\section{Issues of strongly warped KKLT}

The KKLT scenario [22] has been proposed to be a controlled set-up of string moduli stabilization that eventually leads to a dS minimum. It is not a fully fledged string theory construction but rather consists of a number of constituents that were argued to be generically present in consistent string models, working together in an intricate manner to give dS vacua.

Here we do not review the full KKLT scenario, but rather remind the reader of the essential steps. For more details we refer to the literature. One is working in an effective 4D supergravity theory that results from a compactification of the type IIB superstring with fluxes, branes and orientifold planes. Then one proceeds in three steps:

1. Stabilize the complex structure and axio-dilaton moduli via three-form fluxes in a no-scale non-supersymmetric Minkowski minimum with $W_{0} \ll 1$. 
2. Consider the effective theory of the light Kähler modulus described by a Kähler potential and superpotential

$$
K=-3 \log (T+\bar{T}), \quad W=W_{0}+A e^{-a T} .
$$

The non-perturbative effect conspires with the tiny value of $W_{0}$ to stabilize $T$ in a supersymmetric AdS minimum.

3. Uplift the minimum to dS via $\overline{D 3}$ branes at the tip of a warped throat.

This set-up has been scrutinized from various sides in the past. First the validity of step 3 was questioned, namely whether an $\overline{D 3}$-brane at the tip of a warped throat is really a stable configuration (see [48] for a review, and [24-26] for recent developments). Moreover, it has been questioned whether the 4D description of the KKLT AdS minimum does really uplift to a full 10D solution of string theory $[8,49-56]$. More recently, there were also growing concerns even about step 1.

One needs $W_{0} \ll 1$ for the KKLT construction ${ }^{6}$, so this should better not be in the swampland. Recently, in the large complex structure regime, a mechanism has been described that gives $W_{0}=0$ at leading order where subleading (instanton like) terms provide the stabilization of a final light complex structure modulus [58]. This leads to exponentially small values of $W_{0}$. Clearly, for the later uplift one actually needs a similar controllable mechanism [59] close to a conifold point in the complex structure moduli space where large warping can occur.

Relatedly, for the uplift one has to invoke an effective action that is valid in the strongly warped regime. Based on earlier work [60], this question has been addressed recently [24-27] and in the following we will review some of the main results, in particular of [1]. For more details we refer the reader to the original paper ${ }^{7}$.

\subsection{Effective action in the strongly warped regime}

Strongly warped throats can develop close to a conifold point $Z=0$ in the complex structure moduli space. The ultimate question is whether the effective action for the conifold modulus $Z$ is really under control.

There exists a proposal for the effective action of the conifold modulus $Z$ and the warped volume modulus $\mathscr{V}_{w}$ in the strongly warped regime [60]. This is supposed to be valid at a point very close to a conifold singularity where warping effects become relevant. At such points the geometry develops a long throat, at the tip of which a three-cycle $A$ becomes very small. The corresponding complex structure modulus is defined as $Z=\int_{A} \Omega_{3}$, where the physical size of the 3cycle $A$ is $\operatorname{Vol}(A)=\mathscr{V}^{\frac{1}{2}}|Z|$. Therefore, in the regime $\mathscr{V}|Z|^{2} \gg 1$ warping can be neglected whereas for $\mathscr{V}|Z|^{2} \ll 1$ it is substantial. Moreover, such a warped throat can be supported by turning on three-form fluxes $M=\frac{1}{(2 \pi)^{2} \alpha^{\prime}} \int_{A} F_{3}$ and $K=\frac{1}{(2 \pi)^{2} \alpha^{\prime}} \int_{B} H_{3}$.

Locally this deformed conifold geometry is described by a Klebanov-Strassler (KS) throat [64], for which the metric is explicitly known. One can think of the total geometry as such a

\footnotetext{
${ }^{6}$ It was recently argued that large $W_{0}$ values can also provide the supergravity potential with a dS minimum [57]. It is however not clear that this supergravity solution uplifts to a solution of string theory.

${ }^{7}$ For related recent work on analyzing warped throats and their relation to swampland conjectures see [61-63].
} 
KS throat of length $y_{\mathrm{UV}}$ glued to the bulk Calabi-Yau manifold. The warp factor along the throat direction $y$ is given as

$$
e^{-4 A(y)} \approx \frac{g_{s} M^{2}}{\left(\mathscr{V}_{w}|Z|^{2}\right)^{\frac{2}{3}}} \mathscr{I}(y)
$$

where $\mathscr{V}_{w}|Z|^{2} \ll 1$ as mentioned above and $\mathscr{I}(y)$ is an explicitly known function in the KS solution. We have depicted a sketch of the warped geometry in figure 1.

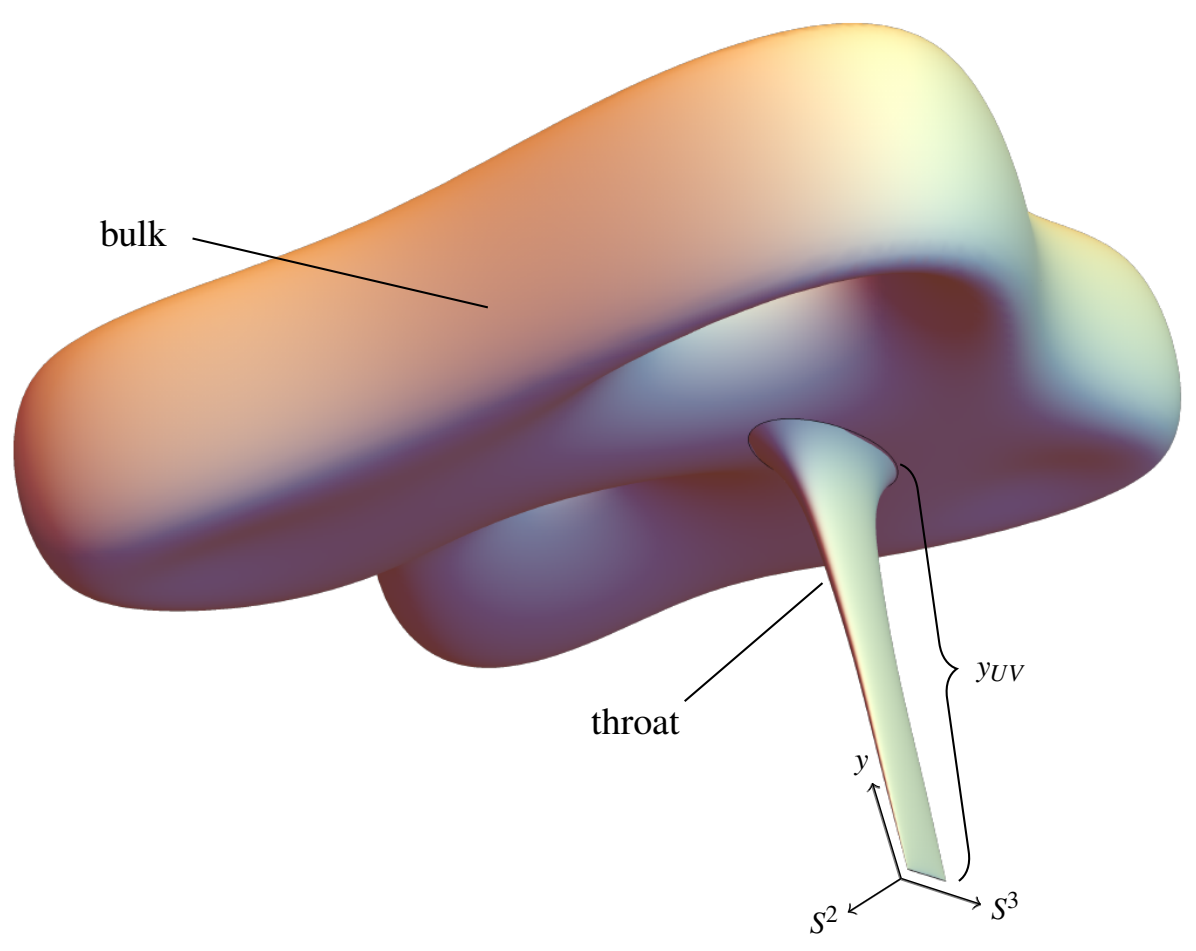

Figure 1: A sketch of a Calabi-Yau with a KS-throat. At the tip of the throat the $S^{2}$ shrinks to zero size while the $S^{3}$ remains finite. $y_{U V}$ marks the cutoff where the throat meets the bulk.

Now, it is assumed that all other complex structure moduli measuring the size of the bulk geometry are stabilized by fluxes at a larger mass scale so that the lightest moduli are $Z$ and the overall Kähler modulus $T$. It turns out that the relevant parameters are $\left\{g_{s}, M, y_{\mathrm{UV}}\right\}$. To be in the large radius regime they need to satisfy $1 \ll g_{s} M^{2}$ and $1 \ll g_{s} M^{2} y_{\mathrm{UV}}^{2}$. It was shown in [60] that the Kähler potential for $Z$ and $T$ reads

$$
K=-3 \log (T+\bar{T})+\frac{c^{\prime} g_{s} M^{2}|Z|^{\frac{2}{3}}}{(T+\bar{T})}+\ldots
$$

featuring a no-scale structure (up to $O\left(g_{s} M^{2}\right)$ ). The three-form flux induced superpotential for the conifold modulus $Z$ reads

$$
W=w_{0}-\frac{M}{2 \pi i} Z \log Z+i K S Z+O(Z)
$$


where as above $M, K$ denote the fluxes through the $A, B$ cycles and $w_{0}$ denotes a constant contribution to the superpotential. This will in general be a non-zero complex number and as mentioned, it would be interesting to generalize the mechanism for $W_{0} \ll 1$ of [58] to the conifold regime. Selfconsistently, the above supergravity model fixes the conifold modulus at the exponentially small value

$$
\left|Z_{0}\right| \sim \exp \left(-\frac{2 \pi K}{g_{s} M}\right)
$$

and its mass turns out to be

$$
m_{Z}^{2} \simeq \frac{1}{g_{s} M^{2}}\left(\frac{|Z|}{\mathscr{V}_{w}}\right)^{\frac{2}{3}} M_{\mathrm{pl}}^{2} \simeq \frac{\left(\mathscr{V}_{w}|Z|^{2}\right)^{\frac{1}{3}}}{g_{s}^{3 / 2} M^{2}} M_{\mathrm{s}}^{2}
$$

This nicely shows that in the regime of strong warping $\mathscr{V}_{w}|Z|^{2} \ll 1$ one indeed finds $m_{Z}<M_{s}$. Moreover, we note that this mass is exponentially small so the question arises whether it is really heavier than the Kähler modulus that is stabilized in step 2.

Computing the scalar potential and adding the contributions of an $\overline{D 3}$ brane at the tip of the throat one obtains

$$
V_{\mathrm{tot}}=\frac{9}{2 c^{\prime} M^{2}} \frac{|Z|^{\frac{4}{3}}}{\operatorname{Re}(T)^{2}}\left[\left(\frac{M}{2 \pi} \log |Z|+\frac{K}{g_{s}}\right)^{2}+\frac{c^{\prime} c^{\prime \prime}}{g_{s}}\right] .
$$

One observes that the three-form flux and the $\overline{D 3}$ brane contribution scale in the same way with the moduli $Z$ and $T$. Therefore, it is not justified to first integrate out $Z$ and then perform the uplift. Plugging in concrete numbers for the constants $c^{\prime}, c^{\prime \prime}$, it was shown [24] that the minimum continues to exist only for $\sqrt{g_{s}} M>6.8$. Thus, in the perturbative regime $\left(g_{s}<1\right)$ one needs a large flux which could be in conflict with tadpole cancellation.

Now, let us consider step 2 of KKLT and add the non-perturbative term $W_{T}=A \exp (-a T)$. Let us assume that one can fix the other complex structure moduli and the axio-dilaton such that $w_{0}$ and $Z$ are of the same order. More on this will be reported in [59]. Then the scale of the superpotential in the minimum is

$$
\left|W_{0}\right| \sim\left|Z_{0}\right| \sim \exp \left(-\frac{2 \pi K}{g_{s} M}\right)
$$

and therefore also exponentially small. As a consequence, in the KKLT AdS minimum, the mass of $T$ scales as

$$
m_{T}^{2} \sim \frac{W_{0}^{2}}{\mathscr{V}_{w}^{2 / 3}} M_{\mathrm{pl}}^{2}<m_{Z}^{2}
$$

Therefore, self-consistently the mass of the Kähler modulus is still lighter than the mass of the conifold modulus. It seems that everything is fine once $\left|w_{0}\right| \sim|Z|$ can indeed be realized in the string landscape. 


\subsection{KK modes and emergence}

However, there is one issue that requires closer inspection, namely that in the strongly warped throat geometry one direction (namely $y$ along the throat) becomes much larger than the other bulk directions. Therefore, one is dealing with a highly non-isotropic geometry that could support very light Kaluza-Klein modes [65-68]. We will see that such modes indeed exist and that, reminiscent of infinite distance limits their mass scale is related to the distance of the conifold point in the complex structure moduli space via emergence ${ }^{8}$.

To estimate the mass scale of the KK modes one needs to solve the six-dimensional warped Laplace equation

$$
e^{4 A(y)} \nabla^{m} \nabla_{m} \chi(y)-m^{2} e^{2 A(y)} \chi(y)=-m_{\mathrm{KK}}^{2} \chi(y) .
$$

In the regime close to the tip of the throat one can use the explicitly known KS solution and it has been shown in [1] that there exist light KK modes with mass

$$
m_{\mathrm{KK}}^{2}=c \frac{n^{2}}{y_{\mathrm{UV}}^{2}} m_{Z}^{2}
$$

With respect to $g_{s}, M$ and $\left(\mathscr{V}_{w}|Z|^{2}\right)$, this scales precisely as $m_{Z}^{2}$. The requirement that the description in terms of a warped throat is consistent constrains the UV cutoff $y_{\mathrm{UV}}$ to $y_{\mathrm{UV}}>1$. In this case there are a finite number of KK modes which are lighter than the conifold modulus. Thus the description in terms of the effective action could break down.

Remarkably, the existence and mass scale of these KK modes is consistent with the picture of emergence of the field space metric [19], even though here the conifold locus is at finite distance in the complex structure moduli space. Indeed, the distance from the conifold locus in the warped case in terms of the $Z$ modulus is given by

$$
\Phi=\int d \zeta \sqrt{G_{Z \bar{Z}}} \sim\left(\frac{|Z|}{\mathscr{V}_{w}}\right)^{\frac{1}{3}} .
$$

Guided by the emergence proposal, one can determine the cut-off $\Lambda$ of the effective theory. For that purpose, one assumes that in the regime $g_{s} M^{2} \gg 1$ the tower of KK modes

$$
\Delta m \approx \frac{1}{\sqrt{g_{s} M^{2}} y_{\mathrm{UV}}}\left(\frac{|Z|}{\mathscr{V}_{w}}\right)^{\frac{1}{3}} M_{\mathrm{pl}}
$$

is lighter than the cut-off. When these are integrated out, the field space metric should get a oneloop correction proportional to the tree-level result. Assuming $\operatorname{deg}(n)=1$ one finds

$$
g_{\mathrm{Z}}^{1-\text { loop }} \sim N_{\mathrm{sp}}^{3} \frac{1}{g_{s} M^{2} y_{\mathrm{UV}}^{2}} \frac{1}{\left(\mathscr{V}_{w}|Z|^{2}\right)^{2 / 3}} .
$$

Matching this with the tree level result

$$
g_{Z \bar{Z}}^{\text {tree }} \sim \frac{g_{s} M^{2}}{\left(\mathscr{V}_{w}|Z|^{2}\right)^{2 / 3}},
$$

\footnotetext{
${ }^{8}$ In a similar spirit, in [69] the WGC, the swampland distance conjecture and emergence have been analyzed for type IIB orientifolds with closed string abelian gauge fields.
} 
one can fix the species scale $N_{\text {sp }}$, i.e. the number of KK-states below the cutoff, as

$$
N_{\mathrm{sp}} \sim\left(g_{s} M^{2} y_{\mathrm{UV}}\right)^{2 / 3}
$$

Then, as one knows the spacing and number of modes below the cutoff, one finds for the cut-off

$$
\Lambda \sim \sqrt{g_{s} M^{2}}\left(\frac{|Z|}{\mathscr{V}_{w}}\right)^{\frac{1}{3}} M_{\mathrm{pl}} .
$$

Intriguingly, this cutoff equals the mass of a D3-brane wrapping the A-cycle $S^{3}$ which vanishes at the conifold. Indeed, it is well known that the conifold singularity in the complex structure moduli space arises from integrating out precisely such a D3-brane [70]. Including the warp factor, one can determine the mass of the wrapped D3-brane as

$$
m_{\mathrm{D} 3}^{2} \sim g_{s}^{\frac{1}{2}} M^{2}\left(\mathscr{V}_{w}|Z|^{2}\right)^{\frac{1}{3}} M_{s}^{2} \sim g_{s} M^{2}\left(\frac{|Z|}{\mathscr{V}_{w}}\right)^{\frac{2}{3}} M_{\mathrm{pl}}^{2} \sim \Lambda^{2} .
$$

How does this cut-off relate to the earlier cut-off $y_{\mathrm{UV}}$ of the throat length? An upper bound for $y_{\mathrm{UV}}$ can be obtained by demanding the throat volume $\mathscr{V}_{w}^{\text {throat }}$ to be smaller than the volume of the whole Calabi Yau, $\mathscr{V}_{w}^{\text {throat }}<\mathscr{V}_{w}$. At leading order in the Klebanov-Strassler solution this constraint leads to

$$
y_{\mathrm{UV}} \lesssim 3 \log \left(M_{\mathrm{pl}} / \Lambda\right)
$$

relating the two introduced cut-offs $y_{\mathrm{UV}}$ and $\Lambda$ in a simple manner.

In summary, the following picture for the mass scales in the effective theory of the warped throat arises:

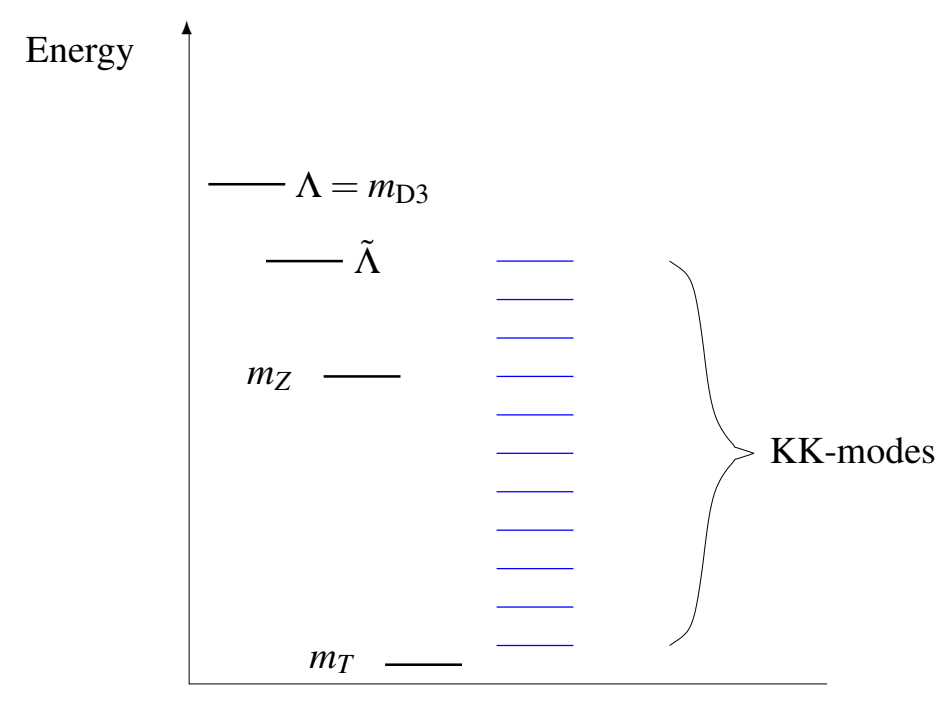

Figure 2: Mass scales in the warped throat in the regime $y_{\mathrm{UV}}>1$.

As in the swampland distance conjecture, there are KK-modes which are lighter than the cut-off so that one expects the effective theory to be outside of the controlled regime. But the emergence 
proposal implies that the corrections induced by these modes are proportional to the tree level result, only changing numerical factors. It is not clear to us what this extra special structure implies for the reliability of the "naive" effective action. Could this not indicate that it is possible that the "naive" effective action is much better than one might initially think? For sure the effect of including the extra light KK modes in the action will not change the functional dependence of the kinetic term of the modulus $Z$, even though there might be other more drastic effects. Unfortunately, we cannot give a conclusive answer to the question of the reliability of the effective action and leave it for future work.

\section{Quantum Log-Corrections to Swampland Conjectures}

Having reviewed recent work on the effective theory in the warped throat, let us now focus on the KKLT AdS minimum, while assuming that $W_{0}$ can indeed be tuned to exponentially small values. Since the masses of the moduli $T, Z$ parametrically satisfy $m_{T}<m_{Z}$, we are confident that the AdS minimum where $T$ is stabilized by a non-perturbative contribution, indeed belongs to the string theory landscape. In the following we will review the arguments [2] suggesting that log-corrections to the AdS/moduli scale separation and the AdS distance conjecture occur.

We have already seen in section 2 that the quantum generalization of the dS swampland conjecture, namely the TCC, also included such log-corrections. In this case, the bound on the life-time of a dS phase got weakened (2.6). Therefore, it is natural to wonder whether a similar relaxation of the bounds also occurs for the AdS conjectures.

\subsection{KKLT and the AdS-moduli scale separation conjecture}

Recalling once more the KKLT construction, the AdS vacuum occurs in its second step. Assuming real values for $W_{0}, A \in \mathbb{R}$ with $W_{0} A<0$, the scalar potential after freezing the axion at $\theta=0$ reads

$$
V_{\mathrm{KKLT}}=\frac{a A^{2}}{6 \tau^{2}} e^{-2 a \tau}(3+a \tau)+\frac{a A W_{0}}{2 \tau^{2}} e^{-a \tau}
$$

In order to determine the position of the supersymmetric AdS vacuum, one needs to solve the transcendental equation

$$
A(2 a \tau+3)=-3 W_{0} e^{a \tau}
$$

leading to a negative cosmological constant

$$
\Lambda=-\frac{a^{2} A^{2}}{6 \tau} e^{-2 a \tau}
$$

Concerning the AdS distance conjecture, it is clear that the $\Lambda \rightarrow 0$ limit occurs for $\tau \rightarrow \infty$, i.e. at infinite distance in the moduli space. One can easily compute the mass of the $\tau$ modulus in the minimum $V_{0}$ as

$$
m_{\tau}^{2}=\left.\frac{1}{2} K^{\tau \tau} \partial_{\tau}^{2} V\right|_{V_{0}}=\frac{a^{2} A^{2}}{9 \tau}\left(2+5 a \tau+2 a^{2} \tau^{2}\right) e^{-2 a \tau}
$$


This is the modulus relevant for the AdS-moduli scale separation conjecture. Even taking into account the conifold modulus and the additional KK modes of the throat, the Kähler modulus remains the lightest. Plugging in equation (5.3), we can express it as

$$
m_{\tau}^{2}=-\frac{2}{3}\left(2+5 a \tau+2 a^{2} \tau^{2}\right) \Lambda
$$

It is clear that the $\tau$-dependent terms inside the parenthesis violate the bound (2.1), so in the controlled regime $a \tau \gg 1$ scale separation is possible to some extent. Expressing the above equation only in terms of $\Lambda$ and neglecting $\log \log$-corrections, one can write

$$
m_{\tau}^{2}=-\left(c_{2}^{2} \log ^{2}(-\Lambda)+c_{1} \log (-\Lambda)+c_{0}\right) \Lambda .
$$

This suggests to declare a quantum version of the AdS/moduli scale separation conjecture taking the form

$$
m_{\text {mod }} R_{\mathrm{AdS}} \leq c \log \left(R_{\mathrm{AdS}} M_{\mathrm{pl}}\right)
$$

where we have reintroduced the Planck-scale. This gives a weaker bound on the combination on the left-hand side allowing a log-type scale separation between the lightest moduli mass and the AdS scale. Moreover, in the $M_{\mathrm{pl}} \rightarrow \infty$ limit where gravity decouples, the quantum version of the conjecture becomes trivial, as expected.

\subsection{KKLT and the AdS distance conjecture}

Let us now discuss the AdS distance conjecture, according to which in the $\Lambda \rightarrow 0$ limit there must appear a tower of states with masses $m_{\text {tower }}=c|\Lambda|^{\alpha}$. In the original paper [9] it was not clear whether the KKLT AdS vacuum abides by this conjecture. The reason is that it is not trivial to identify which is the tower that should behave in the above way. The tower that would immediately come to mind is the Kaluza-Klein tower. Usually, the mass scale of the KK states on an isotropic manifold is estimated as the inverse of the relevant length scale. For the KKLT case, that would give the estimate

$$
m_{\mathrm{KK}} \sim \frac{1}{\tau}
$$

However, this does not exhibit the behaviour expected by the ADC, regardless of the value of the exponent in the conjecture. In fact, this mass scale is exponentially larger than $|\Lambda|^{\alpha}$ for any $\alpha$. This exponential mismatch however should not necessarily be interpreted as a failure of the conjecture, but rather as a lack of knowledge of the relevant tower of states or the reliability of the naive estimate (5.8).

Concerning the latter possibility, we have already discussed in section 3 that the solution to the actual eigenvalue problem (3.12) can have important effects. Coming to the other point, it is not necessary that the naive estimate for the KK scale (5.8) really gives the mass of all possible KK modes. This is even evident from the example of a non-isotropic two-dimensional torus with a short and a long circle. The long circle gives rise to a KK tower whose mass is lower than (5.8). 
The strongly warped throat geometry is precisely of this type and there we have identified already a tower of lighter KK modes localized close to the tip of the throat, scaling as

$$
m_{\mathrm{KK}}^{2} \sim \frac{1}{y_{\mathrm{UV}}^{2}}\left(\frac{Z}{\mathscr{V}_{w}}\right)^{\frac{2}{3}} .
$$

In the limit where the throat just fits into the warped CY we can approximate

$$
y_{\mathrm{UV}} \sim-\log \left(|Z| / \tau^{\frac{3}{2}}\right) \sim \tau
$$

so that there actually exists an exponentially light KK tower. Its mass scale can be expressed at the KKLT AdS minimum as

$$
m_{\mathrm{KK}}^{2} \sim \frac{1}{\tau^{2}} \frac{e^{-\frac{2}{3} a \tau}}{\tau^{\frac{1}{3}}} \sim \frac{1}{\log ^{2}(-\Lambda)}|\Lambda|^{\frac{1}{3}} .
$$

One realizes that this behavior, up to the log-corrections, satisfies the ADC for $\alpha=1 / 6$. Since $\alpha<1 / 2$, one has scale separation between the AdS scale and the size of the internal geometry.

\subsection{KKLT and emergence}

Let us now comment on an intriguing connection to the emergence proposal. Recall from section 4 that the subleading term in the Kähler potential

$$
K=-3 \log (T+\bar{T})+c \frac{|Z|^{\frac{2}{3}}}{(T+\bar{T})}
$$

is corrected by the tower of red-shifted KK modes with mass scale (5.9) at the tip of the throat. For the species scale one finds

$$
\Lambda_{\mathrm{sp}}^{3} \sim \frac{|Z|}{\tau^{\frac{3}{2}} y_{\mathrm{UV}}} M_{\mathrm{pl}}^{3},
$$

where $y_{\mathrm{UV}}$ is given in large throat limit by (5.10). Since the first term in $K$ is also present in the unwarped case, from the perspective of the emergence proposal one expects it to emerge from integrating out the tower of bulk KK modes $\Delta m_{\mathrm{KK}, \mathrm{h}} \sim 1 / \tau$. Stabilizing $\tau$ via KKLT and as usual assuming

$$
|Z| \sim\left|W_{0}\right| \sim \tau e^{-a \tau}
$$

the species scale can be expressed as

$$
\Lambda_{\mathrm{sp}}^{3} \sim \frac{e^{-a \tau}}{\tau^{\frac{3}{2}}} M_{\mathrm{pl}}^{3} \sim \Lambda_{\mathrm{SQCD}}^{3},
$$

where $\Lambda_{\mathrm{SQCD}}$ is the dynamically generated mass scale of the confining SYM theory which undergoes gaugino condensation,

$$
\Lambda_{\mathrm{SQCD}}^{3}=e^{-a / g^{2}} M^{3} .
$$

Here the UV cut-off $M$ of the gauge theory is in turn not the Planck scale but rather $M \sim g M_{\mathrm{pl}}$ as implied by the magnetic weak gravity conjecture [71]. We think that this match with the expected cut-off of the KKLT model provides a compelling result as it relies on a non-trivial combination of results from section 4 with swampland conjectures. 


\subsection{KKLT and the dS swampland conjectures}

It was already pointed out in [17] that the uplifted KKLT dS minimum does violate not only the $\mathrm{dS}$ swampland conjecture but also the trans-Planckian censorship conjecture. The reason is simply that the life-time of the dS vacuum as computed via the Coleman-De Luccia bubble nucleation [22] comes out exponentially large and violates (2.6).

Let us therefore look for a dS saddle point. Indeed, it is known [72] that including the $\alpha^{\prime}$ correction to the Kähler potential

$$
K=-2 \log \left((T+\bar{T})^{\frac{3}{2}}+\frac{\xi}{2}\right), \quad W=W_{0}+A e^{-a T}
$$

for $\xi>0$ leads to a dS saddle point at large values of $\tau$. This is shown in figure 3 .
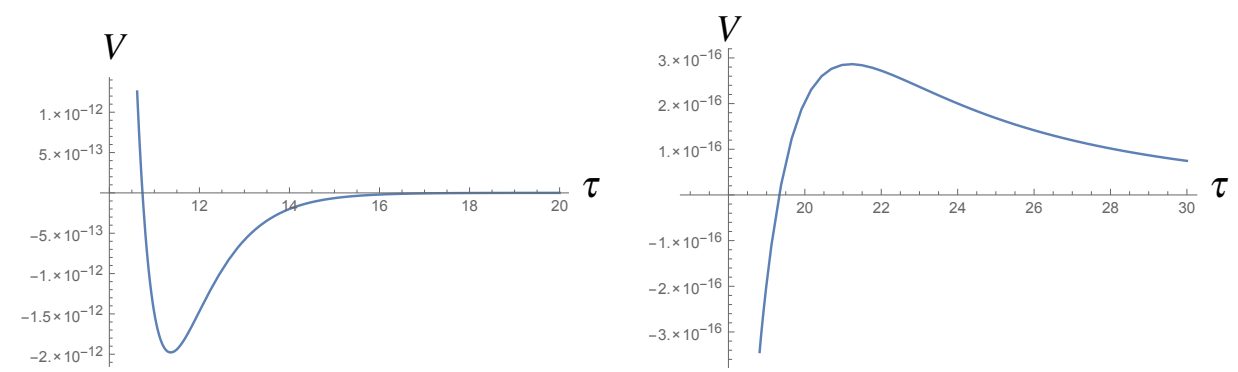

Figure 3: The $\alpha^{\prime}$ corrected KKLT potential (after integrating out the axion) for $W_{0}=10^{-4}, A=-1, a=1$ and $\xi=1$ : The figure on the left shows the existence of the AdS minimum, while the one on the right reveals the existence of a dS saddle point at a larger value of $\tau$.

In the large $a \tau$ regime and after minimization with respect to the axion, the scalar potential for $\tau$ can be approximated as

$$
V=\frac{3 \xi\left|W_{0}\right|^{2}}{64 \sqrt{2} \tau^{\frac{9}{2}}}-\frac{a\left|A W_{0}\right|}{2 \tau^{2}} e^{-a \tau}+\ldots
$$

where we neglected subleading terms. The position $\tau_{0}$ of the saddle point is given by the solution of the transcendental relation

$$
\left|W_{0}\right|=\frac{64 \sqrt{2} a|A|}{27 \xi} \tau_{0}^{\frac{5}{2}}\left(2+a \tau_{0}\right) e^{-a \tau_{0}} .
$$

Working in the regime $a \tau \gg 1$, the value of the potential at the dS saddle point and its tachyonic mass can be determined as

$$
V_{0} \approx \frac{64 \sqrt{2}}{243 \xi} a^{4}|A|^{2} \tau_{0}^{\frac{5}{2}} e^{-2 a \tau_{0}}, \quad m_{0}^{2} \approx-\frac{64 \sqrt{2}}{81 \xi} a^{5}|A|^{2} \tau_{0}^{\frac{7}{2}} e^{-2 a \tau_{0}}
$$

so that

$$
-\frac{m_{0}^{2}}{V_{0}} \approx 3 a \tau_{0} \approx \frac{3}{2} \log \left(\frac{1}{V_{0}}\right)
$$


In the regime of control $a \tau \gg 1$, this clearly satisfies the second condition in the refined dS swampland conjecture (2.3). Moreover, by using $V_{0} \sim H^{2}$, the life-time of the dS saddle point can be expressed as

$$
T \sim \frac{1}{H \log \left(\frac{M_{\mathrm{pl}}}{H}\right)}<\frac{1}{H}<\frac{1}{H} \log \left(\frac{M_{\mathrm{pl}}}{H}\right) .
$$

Therefore, it satisfies the TCC and also contains a $\log H$-correction. However, in this case the $\log$ factor shortens the life-time and does not extend it as for the upper bound in the TCC (2.6). This simple example shows that in non-perturbative KKLT like vacua indeed log-corrections appear, but they go in the opposite direction than required for a saturation of the TCC bound.

\section{Conclusions}

In this article we have reviewed a couple of issues that arose when confronting the KKLT scenario with the swampland conjectures. One not yet excluded possibility could still be that the KKLT scenario as a whole lies in the swampland of string theory and only combines string theory ingredients in a manner that can never be achieved in a fully fledged string theory construction.

However, we think that so far the KKLT scenario and in particular the initial AdS minimum has survived a couple of attacks, that even shed some new light onto the many delicate issues hidden in this simple initial idea. Examples are the questions whether $W_{0} \ll 1$ is possible in a controlled manner or whether KKLT can be controlled in a strongly warped throat geometry. In the latter respect, we pointed out that a destabilization of the uplifted dS minimum can occur (for $\sqrt{g_{s}} M<6.8$ ) and that there exists a tower of highly red-shifted KK modes localized near the tip of the throat that could potentially spoil the control over the utilized low energy effective action.

Assuming and being confident that at least the AdS minimum (prior to uplift) à la KKLT does exist, we reviewed how this relates to the "classical" AdS and dS swampland conjectures, motivating the introduction of quantum log-factors at various places. Concretely, we have proposed corrections to the AdS/moduli scale separation conjecture as well as the AdS distance conjecture. Both these quantum generalizations lead to weaker bounds than the classical conjectures. In particular, a log-type scale separation is allowed between the AdS space and the lightest modulus.

Viewing KKLT from the emergence perspective, through a remarkable interplay of swampland conjectures we found that the natural species scale cut-off of KKLT coincides with the gaugino condensate scale of the implicitly assumed confining gauge theory $\Lambda_{\mathrm{sp}} \sim \Lambda_{\mathrm{SQCD}}$. Finally, as a new result we found that after including $\alpha^{\prime}$ corrections to the Kähler potential the appearing dS saddle point has a life-time that is even smaller than the bound from the dS swampland conjecture. Hence, in this case the log-corrections correction do not weaken the bound towards saturating the bound from TCC.

\section{References}

[1] R. Blumenhagen, D. Kläwer and L. Schlechter, JHEP 1905, 152 (2019) [arXiv:1902.07724 [hep-th]]. 
[2] R. Blumenhagen, M. Brinkmann and A. Makridou, JHEP 2002, 064 (2020) [JHEP 2020, 064 (2020)] [arXiv:1910.10185 [hep-th]].

[3] C. Vafa, hep-th/0509212.

[4] H. Ooguri and C. Vafa, Nucl. Phys. B 766, 21 (2007) [hep-th/0605264].

[5] T. D. Brennan, F. Carta and C. Vafa, PoS TASI 2017, 015 (2017) [arXiv:1711.00864 [hep-th]].

[6] E. Palti, Fortsch. Phys. 67, no. 6, 1900037 (2019) [arXiv:1903.06239 [hep-th]].

[7] E. Palti, C. Vafa and T. Weigand, arXiv:2003.10452 [hep-th].

[8] F. F. Gautason, V. Van Hemelryck and T. Van Riet, Fortsch. Phys. 67, no. 1-2, 1800091 (2019) [arXiv:1810.08518 [hep-th]].

[9] D. Lüst, E. Palti and C. Vafa, Phys. Lett. B 797, 134867 (2019) [arXiv:1906.05225 [hep-th]].

[10] L. F. Alday and E. Perlmutter, JHEP 1908, 084 (2019) [arXiv:1906.01477 [hep-th]].

[11] A. Kehagias, D. Lüst and S. Lüst, arXiv:1910.00453 [hep-th].

[12] F. F. Gautason, M. Schillo, T. Van Riet and M. Williams, JHEP 1603, 061 (2016) [arXiv:1512.00457 [hep-th]].

[13] G. Obied, H. Ooguri, L. Spodyneiko and C. Vafa, arXiv:1806.08362 [hep-th].

[14] H. Ooguri, E. Palti, G. Shiu and C. Vafa, Phys. Lett. B 788, 180 (2019) [arXiv:1810.05506 [hep-th]].

[15] S. K. Garg and C. Krishnan, JHEP 1911, 075 (2019) [arXiv:1807.05193 [hep-th]].

[16] D. Andriot, Phys. Lett. B 785, 570 (2018) [arXiv:1806.10999 [hep-th]].

[17] A. Bedroya and C. Vafa, arXiv:1909.11063 [hep-th].

[18] B. Heidenreich, M. Reece and T. Rudelius, Eur. Phys. J. C 78, no. 4, 337 (2018) [arXiv:1712.01868 [hep-th]].

[19] T. W. Grimm, E. Palti and I. Valenzuela, JHEP 1808, 143 (2018) [arXiv:1802.08264 [hep-th]].

[20] B. Heidenreich, M. Reece and T. Rudelius, Phys. Rev. Lett. 121, no. 5, 051601 (2018) [arXiv:1802.08698 [hep-th]].

[21] D. Klaewer and E. Palti, JHEP 1701, 088 (2017) [arXiv:1610.00010 [hep-th]].

[22] S. Kachru, R. Kallosh, A. D. Linde and S. P. Trivedi, Phys. Rev. D 68, 046005 (2003) [hep-th/0301240].

[23] V. Balasubramanian, P. Berglund, J. P. Conlon and F. Quevedo, JHEP 0503, 007 (2005) [hep-th/0502058].

[24] I. Bena, E. Dudas, M. Graña and S. Lüst, Fortsch. Phys. 67, no. 1-2, 1800100 (2019) [Fortsch. Phys. 2018, 1800100] [arXiv:1809.06861 [hep-th]].

[25] I. Bena, A. Buchel and S. Lüst, arXiv:1910.08094 [hep-th].

[26] E. Dudas and S. Lüst, arXiv:1912.09948 [hep-th].

[27] L. Randall, arXiv:1912.06693 [hep-th].

[28] T. W. Grimm, C. Li and I. Valenzuela, arXiv:1910.09549 [hep-th].

[29] D. Junghans, arXiv:2003.06274 [hep-th]. 
[30] G. Buratti, J. Calderon, A. Mininno and A. M. Uranga, arXiv:2003.09740 [hep-th].

[31] F. Marchesano, E. Palti, J. Quirant and A. Tomasiello, arXiv:2003.13578 [hep-th].

[32] O. DeWolfe, A. Giryavets, S. Kachru and W. Taylor, JHEP 0507, 066 (2005) [hep-th/0505160].

[33] M. P. Hertzberg, S. Kachru, W. Taylor and M. Tegmark, JHEP 0712, 095 (2007) [arXiv:0711.2512 [hep-th]].

[34] Y. Akrami, R. Kallosh, A. Linde and V. Vardanyan, Fortsch. Phys. 67, no. 1-2, 1800075 (2019) [arXiv:1808.09440 [hep-th]].

[35] R. R. Caldwell, R. Dave and P. J. Steinhardt, Phys. Rev. Lett. 80, 1582 (1998) [astro-ph/9708069].

[36] P. Agrawal, G. Obied, P. J. Steinhardt and C. Vafa, Phys. Lett. B 784, 271 (2018) [arXiv:1806.09718 [hep-th]].

[37] S. Brahma, Phys. Rev. D 101, no. 4, 046013 (2020) [arXiv:1910.12352 [hep-th]].

[38] D. Andriot, N. Cribiori and D. Erkinger, arXiv:2004.00030 [hep-th].

[39] R. Blumenhagen, PoS CORFU 2017, 175 (2018) [arXiv:1804.10504 [hep-th]].

[40] S. J. Lee, W. Lerche and T. Weigand, JHEP 1810, 164 (2018) [arXiv:1808.05958 [hep-th]].

[41] S. J. Lee, W. Lerche and T. Weigand, arXiv:1904.06344 [hep-th].

[42] S. J. Lee, W. Lerche and T. Weigand, arXiv:1910.01135 [hep-th].

[43] F. Baume, F. Marchesano and M. Wiesner, arXiv:1912.02218 [hep-th].

[44] P. G. Camara, A. Font and L. E. Ibanez, JHEP 0509, 013 (2005) [hep-th/0506066].

[45] F. Marchesano and J. Quirant, JHEP 1912, 110 (2019) [arXiv:1908.11386 [hep-th]].

[46] D. Lust and D. Tsimpis, arXiv:2004.07582 [hep-th].

[47] A. Font, A. Herráez and L. E. Ibáñez, JHEP 2003, 013 (2020) [arXiv:1912.03317 [hep-th]].

[48] U. H. Danielsson and T. Van Riet, Int. J. Mod. Phys. D 27, no.12, 1830007 (2018) [arXiv:1804.01120 [hep-th]].

[49] J. Moritz, A. Retolaza and A. Westphal, Phys. Rev. D 97, no. 4, 046010 (2018) [arXiv:1707.08678 [hep-th]].

[50] R. Kallosh, A. Linde, E. McDonough and M. Scalisi, Fortsch. Phys. 67, no. 1-2, 1800068 (2019) [arXiv:1808.09428 [hep-th]].

[51] R. Kallosh, A. Linde, E. McDonough and M. Scalisi, Phys. Rev. D 99, no. 4, 046006 (2019) [arXiv:1809.09018 [hep-th]].

[52] Y. Hamada, A. Hebecker, G. Shiu and P. Soler, JHEP 1904, 008 (2019) [arXiv:1812.06097 [hep-th]].

[53] Y. Hamada, A. Hebecker, G. Shiu and P. Soler, JHEP 1906, 019 (2019) [arXiv:1902.01410 [hep-th]].

[54] F. Carta, J. Moritz and A. Westphal, JHEP 1908, 141 (2019) [arXiv:1902.01412 [hep-th]].

[55] F. F. Gautason, V. Van Hemelryck, T. Van Riet and G. Venken, arXiv:1902.01415 [hep-th].

[56] I. Bena, M. Graña, N. Kovensky and A. Retolaza, JHEP 1910, 200 (2019) [arXiv:1908.01785 [hep-th]].

[57] A. Linde, arXiv:2002.01500 [hep-th]. 
[58] M. Demirtas, M. Kim, L. Mcallister and J. Moritz, arXiv:1912.10047 [hep-th].

[59] R. Álvarez-García, R. Blumenhagen, M. Brinkmann and L. Schlechter, “work in progress”.

[60] M. R. Douglas, J. Shelton and G. Torroba, arXiv:0704.4001 [hep-th].

[61] R. Blumenhagen, D. Herschmann and F. Wolf, JHEP 1608, 110 (2016) [arXiv:1605.06299 [hep-th]].

[62] A. Hebecker, S. Leonhardt, J. Moritz and A. Westphal, JHEP 1904, 158 (2019) [arXiv:1812.03999 [hep-th]].

[63] G. Buratti, J. Calderón and A. M. Uranga, JHEP 1905, 176 (2019) [arXiv:1812.05016 [hep-th]].

[64] I. R. Klebanov and M. J. Strassler, JHEP 0008, 052 (2000) [hep-th/0007191].

[65] A. R. Frey and A. Maharana, JHEP 0608, 021 (2006) [hep-th/0603233].

[66] C. P. Burgess, P. G. Camara, S. P. de Alwis, S. B. Giddings, A. Maharana, F. Quevedo and K. Suruliz, JHEP 0804, 053 (2008) [hep-th/0610255].

[67] G. Shiu, G. Torroba, B. Underwood and M. R. Douglas, JHEP 0806, 024 (2008) [arXiv:0803.3068 [hep-th]].

[68] S. P. de Alwis, JHEP 1611, 045 (2016) [arXiv:1605.06456 [hep-th]].

[69] M. Enriquez-Rojo and E. Plauschinn, arXiv:2002.04050 [hep-th].

[70] A. Strominger, Nucl. Phys. B 451, 96 (1995) [hep-th/9504090].

[71] N. Arkani-Hamed, L. Motl, A. Nicolis and C. Vafa, JHEP 0706, 060 (2007) [hep-th/0601001].

[72] J. P. Conlon, Int. J. Mod. Phys. A 33, no. 29, 1850178 (2018) [arXiv:1808.05040 [hep-th]]. 